\title{
Phase Space Approach to Laser-driven Electronic Wavepacket Propagation
}

\author{
Norio Takemoto, ${ }^{1}$ Asaf Shimshovitz, ${ }^{1}$ and David J. Tannor ${ }^{1}$ \\ Department of Chemical Physics, Weizmann Institute of Science, 76100 Rehovot, \\ Israel
}

(Dated: 27 October 2018)

We propose a phase space method to propagate a quantum wavepacket driven by a strong external field. The method employs the so-called biorthogonal von Neumann basis recently introduced for the calculation of the energy eigenstates of time-independent quantum systems [A. Shimshovitz and D.J. Tannor, arXiv:1201.2299v1]. While the individual elements in this basis set are time-independent, a small subset is chosen in a time-dependent manner to adapt to the evolution of the wavepacket in phase space. We demonstrate the accuracy and efficiency of the present propagation method by calculating the electronic wavepacket in a one-dimensional soft-core atom interacting with a superposition of an intense, few-cycle, near-infrared laser pulse and an attosecond extreme-ultraviolet laser pulse.

With the emergence of attosecond laser technology, there is the fascinating prospect of observing and controlling the correlated dynamics of multiple electrons on its natural time scale of ten to one hundred attoseconds 2 In order to unravel the complex and sometimes counterintuitive ${ }^{3,4}$ quantum dynamics from the experimental data, and to develop theories that reproduce the essence of the dynamics 5 , an accurate and efficient numerical method to simulate the multi-electron wavepacket dynamics is indispensable.

However, accurate simulation of the electronic dynamics in a high-intensity laser field is a challenging task: the electronic wavepacket is dispersed by the laser field over a wide region of coordinate space while retaining high momentum near the atomic nuclei. Straightforward representation of the wavefunction on a equally-spaced coordinate grid [i.e., a Fourier grid (FG)] requires a large range with a small interval between points. Simulation on such a large grid quickly becomes prohibitive as the number of degrees of freedom (DOF) increases. Even with sophisticated treatments such as multi-configuration timedependent Hartree-Fock (MCTDHF) $\stackrel{6}{\underline{6}}$ simulation of ionization dynamics have been limited to small systems such as the helium atom and the hydrogen molecule. ${ }^{7-12}$

In this article, we present a new approach to solving the time-dependent Schrödinger equation (TDSE), based on a phase space perspective. The resultant propagation method is simple, accurate, stable, and efficient. As a first demonstration, we simulate the electronic wavepacket of a one-dimensional (1D) model atom in the combined fields of a high-intensity near-infrared (NIR) laser pulse and an attosecond extreme-ultraviolet (XUV) laser pulse.

In our approach, we utilize the localized nature of phase space Gaussians to prune the basis. However, a basis set of phase-space localized states is perforce nonorthogonal $^{13}$, and this has created difficulties in previ-

a) The following article has been submitted to the Journal of Chemical Physics. After it is published, it will be found at http://jcp.aip.org/ ous attempts ${ }^{14-19}$ to truncate the basis to cover only the phase space region of dynamics. This long standing problem was solved recently, by the so-called periodic von Neumann ( $\mathrm{pvN}$ ) basis 20.21 The pvN basis is generated from a set of Gaussians whose centers form a finite lattice in phase space. By imposing periodic boundary conditions on these Gaussians, the pvN basis becomes formally equivalent to the simple and accurate $\mathrm{FG}$ representation 22 Then, its biorthogonal basis, the biorthogonal von Neumann (bvN) basis, can be used for a compact representation of a quantum state. This framework was successfully applied to the calculation of quantum energy eigenstates ${ }^{21}$ Here, the framework is extended for solving the TDSE. Our strategy is to keep the individual elements in the basis set time-independent but to truncate the bvN basis in a time-dependent manner. This avoids the problem of a moving basis 15 that can become over-complete as time elapses and can become unstable. In contrast, here we obtain a stable set of linear ordinary differential equations (ODEs) for the expansion coefficients in the truncated bvN basis.

Before explaining the pvN and bvN bases, we review the formalism of the FG basis to establish notation. We write the Fourier pseudo-spectral and spectral bases $^{23-25}$ as $\left\{\left|\theta_{m}\right\rangle\right\}_{m=1, \ldots, N}$ and $\left\{\left|\phi_{m}\right\rangle\right\}_{m=1, \ldots, N}$, respectively. These bases are orthonormal and span the same $N$-D Hilbert space denoted here as $\mathcal{H}$, resolving the identity in $\mathcal{H}$ as

$$
1_{\mathcal{H}}=\sum_{m=1}^{N}\left|\phi_{m}\right\rangle\left\langle\phi_{m}\left|=\sum_{m=1}^{N}\right| \theta_{m}\right\rangle\left\langle\theta_{m}\right| .
$$

The bases $\left\{\left|\theta_{m}\right\rangle\right\}$ and $\left\{\left|\phi_{m}\right\rangle\right\}$ are localized at the FG points $\left\{x_{m}\right\}_{m=1, \ldots, N}$ in the position space and $\left\{p_{m}\right\}_{m=1, \ldots, N}$ in the momentum space, respectively. For any quantum state $|\Psi\rangle \in \mathcal{H},\left\langle\theta_{m} \mid \Psi\right\rangle=\left\langle x_{m} \mid \Psi\right\rangle \sqrt{\Delta x}$ and $\left\langle\phi_{m} \mid \Psi\right\rangle=\left\langle p_{m} \mid \Psi\right\rangle \sqrt{\Delta p}$, where $\Delta x$ and $\Delta p$ are the grid intervals in position and momentum spaces, respectively.

The pvN $\operatorname{basis}^{20,21}\left\{\left|\tilde{g}_{j}\right\rangle\right\}_{j=1, \ldots, N}$ is defined as

$$
\left|\tilde{g}_{j}\right\rangle=\sum_{m=1}^{N}\left|\theta_{m}\right\rangle\left\langle x_{m} \mid g_{j}\right\rangle \sqrt{\Delta x}
$$


where $\left\{\left|g_{j}\right\rangle\right\}_{j=1, \ldots, N}$ are the phase space Gaussians,

$$
\begin{aligned}
\left\langle x \mid g_{j}\right\rangle=\left(\frac{\gamma}{\pi}\right)^{1 / 4} \exp \left(-\frac{\gamma}{2}\left(x-q_{j}\right)^{2}\right. & +\frac{i}{\hbar} p_{j}\left(x-q_{j}\right) \\
& \left.+\frac{i}{2 \hbar} p_{j} q_{j}\right),
\end{aligned}
$$

whose centers $\left\{\left(q_{j}, p_{j}\right)\right\}_{j=1, \ldots, N}$ constitute a finite lattice in the phase space with the unit cell of area $2 \pi \hbar$ and the momentum-to-position aspect ratio $\hbar \gamma$. Note that the number of Gaussians $N$ is the same as the number of FG points used.

The bvN basis $21 \quad\left\{\left|\tilde{b}_{j}\right\rangle\right\}_{j=1, \ldots, N}$ is defined to be biorthogonal (dual) to the pvN basis, i.e., $\left\langle\tilde{b}_{l} \mid \tilde{g}_{j}\right\rangle=\delta_{l j}$. This gives

$$
\left|\tilde{b}_{j}\right\rangle=\sum_{l=1}^{N}\left|\tilde{g}_{l}\right\rangle\left(S^{-1}\right)_{l j},
$$

where $S^{-1}$ is the inverse of the overlap matrix $S_{l j}=$ $\left\langle\tilde{g}_{l} \mid \tilde{g}_{j}\right\rangle=\Delta x \sum_{m=1}^{N}\left\langle g_{l} \mid x_{m}\right\rangle\left\langle x_{m} \mid g_{j}\right\rangle$ of the pvN basis. The pvN and bvN bases span the same Hilbert space $\mathcal{H}$ as $\left\{\left|\phi_{m}\right\rangle\right\}$ and $\left\{\left|\theta_{m}\right\rangle\right\}$, resolving the identity as

$$
1_{\mathcal{H}}=\sum_{j=1}^{N}\left|\tilde{b}_{j}\right\rangle\left\langle\tilde{g}_{j}\left|=\sum_{j=1}^{N}\right| \tilde{g}_{j}\right\rangle\left\langle\tilde{b}_{j}\right| .
$$

Thus, any wavepacket $|\Psi(t)\rangle \in \mathcal{H}$ can be represented as

$$
|\Psi(t)\rangle=\sum_{j=1}^{N}\left|\tilde{b}_{j}\right\rangle\left\langle\tilde{g}_{j} \mid \Psi(t)\right\rangle .
$$

Due to the localized nature of the Gaussians $\left\{\left|g_{j}\right\rangle\right\}$, the magnitude of $\left\langle\tilde{g}_{j} \mid \Psi(t)\right\rangle$ can be extremely small if the corresponding classical system can not reach the phase space region around $\left(q_{j}, p_{j}\right)$. Defining a set $\mathcal{A}$ such that $\left|\left\langle\tilde{g}_{j} \mid \Psi(t)\right\rangle\right|$ is negligible if $j \notin \mathcal{A}$, we can approximate the wavepacket by a subset of the bvN basis as

$$
|\Psi(t)\rangle \approx \sum_{j \in \mathcal{A}}\left|\tilde{b}_{j}\right\rangle c_{j}(t)
$$

where $c_{j}(t):=\left\langle\tilde{g}_{j} \mid \Psi(t)\right\rangle$. Note that the set $\mathcal{A}$ of active indices can be changed in time in order to keep the number $N_{\mathcal{A}}$ of elements in $\mathcal{A}$ small at all time.

By substituting eq. (77) to the TDSE, we obtain a set of linear ODEs for the active bvN coefficients $\left\{c_{j}\right\}_{j \in \mathcal{A}}$,

$$
\frac{d c_{j}}{d t}=-\frac{i}{\hbar} \sum_{l \in \mathcal{A}} \sum_{m \in \mathcal{A}}\left(\Omega^{-1}\right)_{j l}\left\langle\tilde{b}_{l}|H(t)| \tilde{b}_{m}\right\rangle c_{m}(t)
$$

where $\Omega^{-1}$ is the inverse of the overlap matrix $\Omega_{j l}=$ $\left\langle\tilde{b}_{j} \mid \tilde{b}_{l}\right\rangle=\left(S^{-1}\right)_{j l}$ of the bvN basis, and $H(t)$ is the Hamiltonian operator of the system. The overlap and Hamiltonian matrix elements in eq. (8) can be computed simply via the representations in $\left\{\left|\theta_{m}\right\rangle\right\}$ and $\left\{\left|\phi_{m}\right\rangle\right\} \stackrel{21,23}{=}$ The

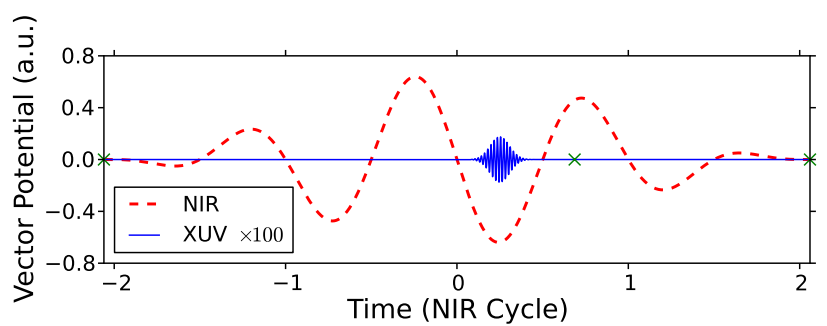

FIG. 1. Vector potentials, $A_{\mathrm{NIR}}(t)$ and $A_{\mathrm{XUV}}(t)$, of the NIR and XUV laser pulses applied to the model 1D atom.

matrix $\Omega^{-1}$ is Hermitian positive-definite, and the elements $\left\{\left\langle\tilde{b}_{l}|H(t)| \tilde{b}_{m}\right\rangle\right\}$ constitute an Hermitian matrix. Therefore, the product of these matrices yields all real eigenvalues ${ }^{26}$, and eq. (8) can be solved stably by many standard numerical algorithms.

To demonstrate the accuracy and efficiency of the present method, we solve eq. (8) for the electronic wavepacket of a $1 \mathrm{D}$ atom in the combined field of NIR and XUV laser pulses. The Hamiltonian of this system is given as

$$
H(t)=H_{0}+V(t),
$$

where $H_{0}$ is the field-free Hamiltonian expressed as

$$
H_{0}=\frac{p^{2}}{2 \mu}-\frac{Q e^{2}}{4 \pi \epsilon_{0} \sqrt{x^{2}+a^{2}}} .
$$

Here $\mu=1$ a.u. is the electron mass, $e=-1$ a.u. is the electron charge, $-Q e=1$ a.u. is the charge of the atomic nucleus, $a=1$ a.u. is the soft-core parameter, and $\epsilon_{0}=1 / 4 \pi$ a.u. is the electric constant. The laserelectron coupling $V(t)$ is, in the velocity gauge,

$$
V(t)=-\frac{e}{\mu}\left[A_{\mathrm{NIR}}(t)+A_{\mathrm{XUV}}(t)\right] p,
$$

where $A_{\mathrm{NIR}}(t)$ and $A_{\mathrm{XUV}}(t)$ are the vector potentials of the NIR and XUV laser pulses, respectively. We used an NIR pulse of wavelength $800 \mathrm{~nm}$, peak intensity $5 \times 10^{13}$ $\mathrm{W} / \mathrm{cm}^{2}$, and duration 1.5 cycles ( $4 \mathrm{fs}$, FWHM of intensity profile). The XUV pulse had wavelength $15 \mathrm{~nm}$, peak intensity $1 \times 10^{12} \mathrm{~W} / \mathrm{cm}^{2}$, and duration 5.0 cycles $(250$ as). The peak of the XUV pulse was delayed from that of the NIR pulse by 0.25 NIR cycles, as shown in Fig. 1.

The initial state was chosen as the ground state of $H_{0}$ with energy eigenvalue -0.66978 a.u., and the wavepacket was propagated from the turn-on of the NIR laser pulse at $t_{\min }$ to its end at $t_{\max }$ by the short-iterative Arnoldi algorithm 27.28 in a $6 \mathrm{D}$ Krylov space with a constant time-step of $\Delta t=0.0379$ a.u. We divided the time span from $t_{\min }$ to $t_{\max }$ into 8 time segments and changed the active set $\mathcal{A}$ from one segment to the next. The number of FG points was $N=4096$, and they were distributed over -750 a.u. $\leq x \leq 750$ a.u. and -8.58 a.u. $\leq p \leq 8.58$ a.u. This phase space rectangle was divided 

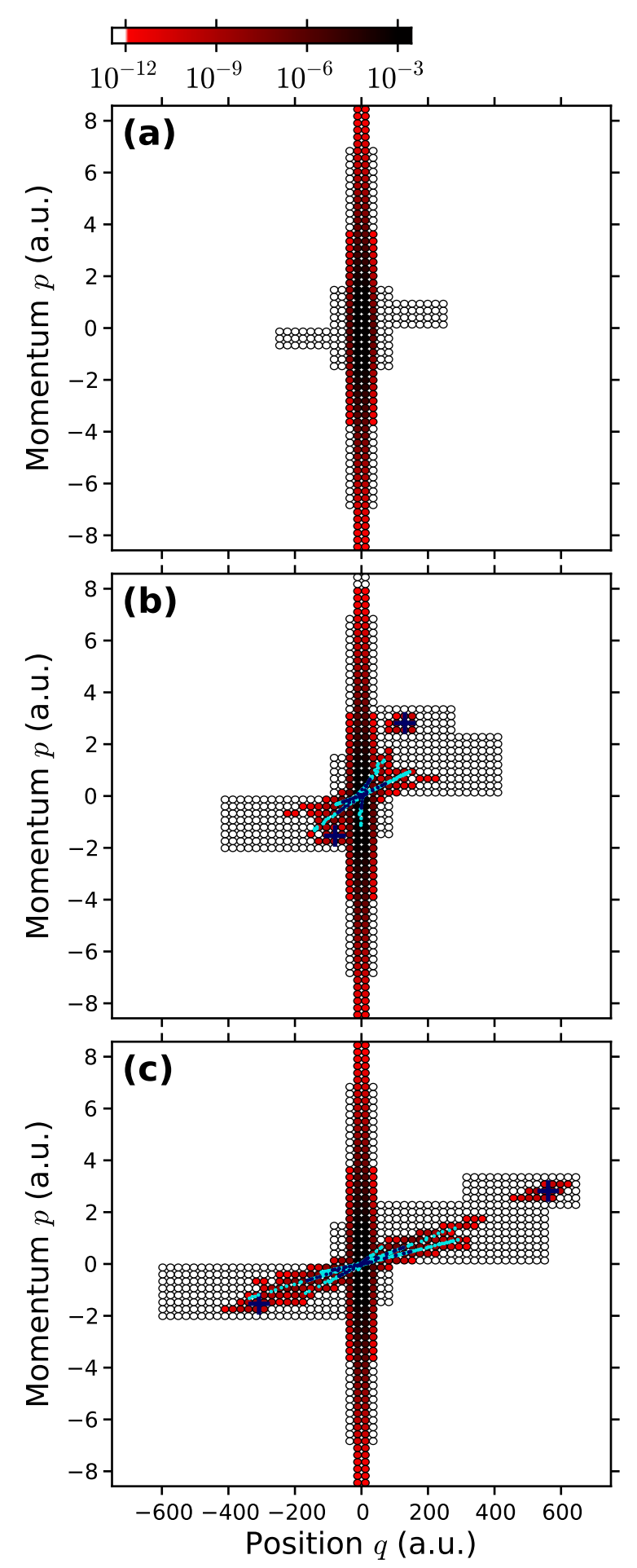

FIG. 2. Snapshots of $\left\{\left|c_{j}\right|^{2}\right\}_{j \in \mathcal{A}}$ shown by the ellipses located at the Gaussian centers $\left\{\left(q_{j}, p_{j}\right)\right\}_{j \in \mathcal{A}}$. The colors of the ellipses indicate the magnitude of $\left|c_{j}\right|^{2}$ according to the scale above the figure. The sequence of dark blue dots represent the simple-man trajectories for direct ionization; the light blue dots represent the rescattered simple-man trajectories. The dark blue + marks represent the simple-man trajectories absorbing one XUV photon in the presence of the NIR field. The snapshots were taken at (a) $t=-2.06$, (b) $t=0.69$, and (c) $t=2.06$ in units of NIR cycles. These times are indicated by the green $\times$ marks in Fig. 1

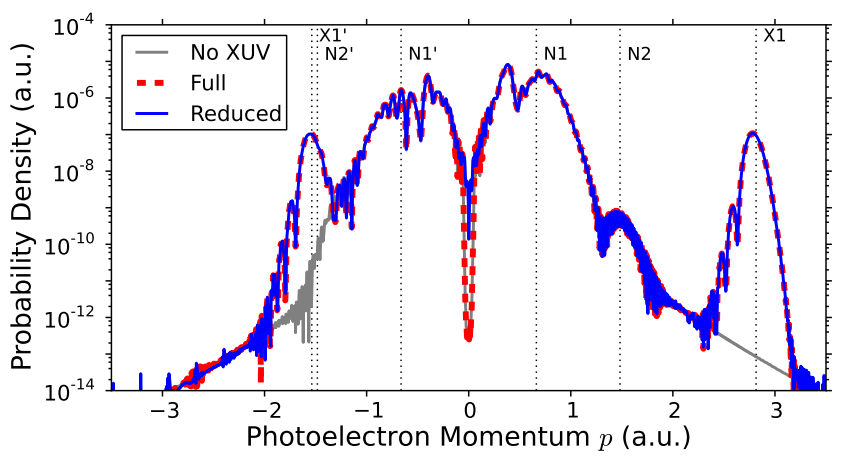

FIG. 3. Comparison of the photoelectron momentum distributions obtained with the reduced bvN basis (blue solid line) and full bvN basis (red dashed line). The momentum distribution from a simulation without the XUV pulse (using the full bvN basis) is also shown (gray solid line). The vertical dashed lines indicate the cut-offs of the direct (N1 and N1') and rescattered (N2 and N2') photoelectrons, as well as the NIR-streaked single-XUV-photon ionization peaks (X1 and $\mathrm{X} 1^{\prime}$ ), estimated by the simple-man model.

into $64 \times 64$ cells of aspect ratio $\hbar \gamma=1.14 \times 10^{-2}$ a.u., each of which contained a Gaussian center $\left(q_{j}, p_{j}\right)$.

In Fig. 2, snapshots of $\left|c_{j}(t)\right|^{2}$ are shown by the color scale of the ellipses located at the active Gaussian centers $\left\{\left(q_{j}, p_{j}\right)\right\}_{j \in \mathcal{A}}$. The outer rectangular boundary of each panel indicates the phase space area corresponding to the Hilbert space $\mathcal{H}$ spanned respectively by the Fourier spectral and pseudospectral bases as well as the full pvN and the full bvN bases. The wavepacket, initially concentrated at the atomic core [Fig. 2(a)], is ionized by the NIR and XUV laser pulses and spreads into parts of the first $(x>0$ and $p>0)$ and third $(x<0$ and $p<0)$ quadrants [Fig. 2(b,c)], but a large area is never accessed. This can be intuitively expected from the classical mechanics, and indeed we see that the wavepacket closely follows the so-called simple-man trajectories ${ }^{\underline{5}}$ (dots and + marks in Fig. 2) which obey the classical Hamiltonian of the same form as eq. (9) with $H_{0}$ replaced by $p^{2} / 2 \mu$. In fact, we chose $\mathcal{A}$ so that the active phase space domain contains these simple-man trajectories (with an additional margin).

In Fig. 3 we compare the photoelectron momentum distributions obtained using the reduced basis of Fig. 2 and the full bvN basis. The excellent agreement between the two results indicates that the present method not only preserves the qualitative features - cut-offs of the direct and rescattered NIR photoelectrons, and the NIRstreaked single-XUV-photon ionization peaks - but also has quantitative accuracy.

The accuracy and the size of the active bvN basis set are expected to be inversely related. However, it is not straightforward to determine the minimal size of the active set that maintains a given accuracy. To seek an upper bound to such an optimal basis size, we carried out simulations with different active sets generated 


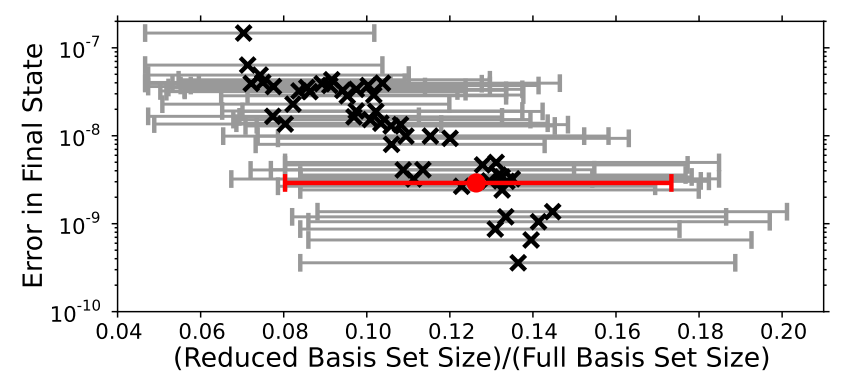

FIG. 4. The error $\epsilon$ as a function of $\left\langle N_{\mathcal{A}}\right\rangle / N$ (black $\times$ marks). The horizontal error bars indicate the range of $N_{\mathcal{A}}(t) / N$ in $t_{\min } \leq t \leq t_{\max }$. The data marked by the red filled circle is from the simulation shown in Figs. 2 and 3 .

by changing the margins around the simple-man trajectories. The error of a simulation was measured by $\epsilon:=\left|\left\langle\Psi^{\text {reduc }}\left(t_{\max }\right) \mid \Psi^{\text {full }}\left(t_{\max }\right)\right\rangle-1\right|$, where $\left|\Psi^{\text {reduc }}\left(t_{\max }\right)\right\rangle$ and $\left|\Psi^{\text {full }}\left(t_{\max }\right)\right\rangle$ are the final states calculated using a reduced and the full bvN bases, respectively. Figure 4 shows the dependence of this error on the basis set size. As the size $N_{\mathcal{A}}$ is time-dependent, and as the computational cost of wavepacket propagation depends on $N_{\mathcal{A}}$ quadratically, we characterized $N_{\mathcal{A}}$ by its rootmean-square, $\left\langle N_{\mathcal{A}}\right\rangle:=\sqrt{\int_{t_{\min }}^{t_{\max }} d t\left[N_{\mathcal{A}}(t)\right]^{2} /\left(t_{\max }-t_{\min }\right)}$, as well as its minimum and maximum values. It can be seen that the bvN basis can be compressed down to $\left\langle N_{\mathcal{A}}\right\rangle / N=0.14$ or possibly further while maintaining the accuracy level at $\epsilon=4 \times 10^{-10}$, and at least to $\left\langle N_{\mathcal{A}}\right\rangle / N=0.08$ for $\epsilon=6 \times 10^{-8}$.

The computational cost of the present method is dominated by the multiplication of the matrix $G_{j m}:=$ $(-i / \hbar) \sum_{l \in \mathcal{A}}\left(\Omega^{-1}\right)_{j l}\left\langle\tilde{b}_{l}|H(t)| \tilde{b}_{m}\right\rangle$ and the vector $c_{m}$ $(j, m \in \mathcal{A})$ which scales as $O\left(N_{\mathcal{A}}^{2}\right)$. There is also initial overhead that scales as $O\left(N^{3}\right)$ originating from the computation of $S_{m j}$ and $\left(S^{-1}\right)_{j l}(m, j=1, \ldots, N ; l \in \mathcal{A})$.

Our method can be extended straightforwardly to systems with multiple DOF $\stackrel{21}{n}$ Defining the number $d$ of DOF, the total number $M$ of FG points increases exponentially as $M=O\left(N^{d}\right)$. In contrast, the number $M_{\mathcal{A}}$ of active bvN basis states for atomic and molecular systems in a laser field is expected to scale more slowly than $M$ since the phase space coordinates are generally corre-

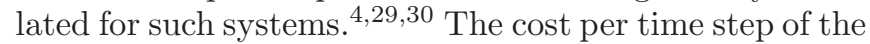
present method goes as $O\left(M_{\mathcal{A}}^{2}\right)$. The initial overhead in calculating $S_{m j}$ and $\left(S^{-1}\right)_{j l}$ stays at $O\left(N^{3}\right)$ because the multi-D Gaussians can be factored into 1D Gaussians 21 . The dominant part in the overhead is now the calculation of the matrix elements for the potential energy operator in the reduced bvN basis, and this goes as $O\left(M M_{\mathcal{A}}^{2}\right)$. For sufficiently large $d$, the present method has the potential to perform better than the popular alternatedirection Crank-Nicolson scheme $\mathrm{e}^{31,32}$ [which costs $O(M)$ per step] and the FG split-operator method 24,25 [which costs $O\left(M \log _{2} N\right)$ per step]. The bvN basis may also be used for the one-body orbitals in MCTDHF as an alterna- tive to the static scaling of the position coordinates $\$, 33,34$ used previously $\underline{\underline{10}}$

In summary, we presented a new method to solve the TDSE based on the bvN basis. Although the basis is time-independent, the active subset is chosen in a timedependent manner. As a first demonstration, we calculated the electronic wavepacket of a 1D atom in the combined fields of intense NIR and attosecond XUV laser pulses. This example demonstrates the high accuracy and efficiency of the method. We are currently working to extend the method to 3D with the aim of ultimately applying it to multi-electron systems in intense and ultrashort laser pulses.

This work was supported by the Minerva Foundation and the Israel Science Foundation under Grant No. $807 / 08$. This research is made possible by the historic generosity of the Harold Perlman family.

${ }^{1}$ T. Popmintchev et al., Nature Photonics 4, 822 (2010).

${ }^{2}$ F. Krausz and M. Ivanov, Rev. Mod. Phys. 81, 163 (2009).

${ }^{3}$ N. Takemoto and A. Becker, Phys. Rev. Lett. 105, 203004 (2010).

${ }^{4}$ M. Odenweller et al., Phys. Rev. Lett. 107, 143004 (2011).

${ }^{5}$ P. B. Corkum, Phys. Rev. Lett. 71, 1994 (1993).

${ }^{6}$ M. Nest, F. Remacle, and R. D. Levine, New J. Phys. 10, 025019 (2008).

${ }^{7}$ J. S. Parker et al., J. Phys. B 36, L393 (2003).

${ }^{8}$ K. Harumiya et al., J. Chem. Phys. 113, 8953 (2000).

${ }^{9}$ J. Zanghellini et al., Laser Phys. 13, 1064 (2003).

${ }^{10}$ T. Kato and H. Kono, J. Chem. Phys. 128, 184102 (2008).

${ }^{11}$ S. X. Hu and L. A. Collins, Phys. Rev. Lett. 96, 073004 (2006).

${ }^{12}$ D. J. Haxton, K. V. Lawler, and C. W. McCurdy, Phys. Rev. A 83, 063416 (2011).

${ }^{13} \mathrm{~F}$. Low, in A Passion for Physics - Essays in Honor of Geoffrey Chew (World Scientific, 1985) pp. 17-22.

${ }^{14}$ D. V. Shalashilin and M. S. Child, J. Chem. Phys. 113, 10028 (2000).

${ }^{15}$ D. V. Shalashilin and M. S. Child, Chem. Phys. 304, 103 (2004).

${ }^{16}$ L. Mauritz Andersson, J. Chem. Phys. 115, 1158 (2001).

${ }^{17}$ M. Satta, E. Scifoni, and F. A. Gianturco, J. Chem. Phys. 118, 2606 (2003).

${ }^{18}$ D. A. McCormack, J. Chem. Phys. 124, 204101 (2006).

${ }^{19}$ Y. Wu and V. S. Batista, J. Chem. Phys. 118, 6720 (2003).

${ }^{20}$ F. Dimler et al., New J. Phys. 11, 105052 (2009).

${ }^{21}$ A. Shimshovitz and D. J. Tannor, arXiv 1201, 2299v1 (2012).

${ }^{22}$ R. Kosloff, in Numerical Grid Methods and Their Application to Schrödinger Equation, edited by C. Cerjan (Kluwer, 1993) pp. 175-194.

${ }^{23}$ C. C. Marston and G. G. Balint-Kurti, J. Chem. Phys. 91, 3571 (1989).

${ }^{24}$ M. Feit, J. Fleck Jr., and A. Steiger, J. Comp. Phys. 47, 412 (1982).

${ }^{25}$ R. Kosloff, J. Phys. Chem. 92, 2087 (1988).

${ }^{26}$ Templates for the Solution of Algebraic Eigenvalue Problems, edited by Z. Bai, J. Demmel, J. Dongarra, A. Ruhe, and H. van der Vorst (SIAM, Philadelphia, 2000) Ch. 5.

${ }^{27}$ W. T. Pollard and R. A. Friesner, J. Chem. Phys. 100, 5054 (1994).

${ }^{28}$ I. Kondov, U. Kleinekathöfer, and M. Schreiber, J. Chem. Phys. 114, 1497 (2001).

${ }^{29}$ A. Staudte et al., Phys. Rev. Lett. 99, 263002 (2007).

${ }^{30}$ J. L. Anchell and J. E. Harriman, J. Chem. Phys. 89, 6860 (1988).

${ }^{31}$ J. Crank and P. Nicolson, Proc. Camb. Phil. Soc. 43, 50 (1947).

${ }^{32}$ D. W. Peaceman and J. H. H. Rachford, J. Soc. Indust. Appl. Math. 3, 28 (1955).

${ }^{33}$ H. Kono et al., J. Comp. Phys. 130, 148 (1997).

${ }^{34}$ I. Kawata and H. Kono, J. Chem. Phys. 111, 9498 (1999). 